\title{
Volatile Sexual Hormones in Mucor mucedo
}

\author{
By D. A. M. MESLAND, J. G. HUISMAN AND H. van DEN ENDE \\ Department of Plant Physiology, University of Amsterdam, \\ Amsterdam, The Netherlands
}

(Received 30 April 1973; revised 9 July 1973)

\section{SUMMARY}

A special technique permitted observation of sexual reactions between mycelia of Mucor mucedo without diffusion contact via the substrate. Morphogenetic phenomena (zygophore formation) appeared to be induced by volatile substances in a sex-specific way. Zygophores, induced via the air, showed chemotropic activity. The volatile stimuli were partly purified by thin-layer chromatography and appeared to have the same $R_{k}$ values as precursors of trisporic acids. Trisporic acids are currently assumed to be responsible for the induction of zygophore formation via the substrate.

\section{INTRODUCTION}

In sexual reproduction in Mucor mucedo and related heterothallic species three reactions can be distinguished (Burgeff, 1924): a 'telemorphotic reaction', which involves the induction of aerial zygophore formation; a 'zygotropic reaction', in which directed growth of zygophores of 'plus' and 'minus' mating partners towards each other is observed; and a 'thigmomorphotic reaction', involving the morphogenetic events which occur after contact of the respective zygophores, such as gametangial fusion and septation.

Zygophore induction. There is evidence that zygophores are induced in a non-specific fashion by the trisporic acids B and C, low molecular weight substances produced by both mating partners and secreted into the culture medium (e.g. Gooday, 1973). In heterothallic species, such as Blakeslea trispora and Mucor mucedo, these hormones ('erogens': Machlis, 1972) are only synthesized when 'plus' and 'minus' mycelia are in continuous diffusion contact via the substrate (van den Ende \& Stegwee, 1971). This synthesis in mated cultures is brought about by the production and secretion into the substrate of precursors of trisporic acids by each strain, and sex-specific conversion to the end products by the mating partners (Sutter, 1970; Sutter, Capage, Harrison \& Keen, 1973). The production of precursors is stimulated by trisporic acids, indicating that hormone synthesis in these fungi is self-amplifying (Bu'Lock \& Winstanley, I97I; Werkman \& van den Ende, 1973). In these studies it became evident that these precursors also are potent zygophore-inducing agents. Unlike the trisporic acids, however, they act in a strictly sex-specific way. They are neutral materials of unknown chemical constitution that were partly purified by thin-layer chromatography (Werkman \& van den Ende, 1973).

Apart from these studies there is a suggestion made by Burgeff (1924) (cf. also Gooday, I973) that zygophore induction in Mucor mucedo could also be mediated by volatile substances. Trisporic acids are not volatile, but the precursors mentioned above might be.

Zygotropic reaction. When 'plus' and 'minus' zygophores are in close proximity, a directed outgrowth towards one another can be observed. It is generally assumed that this reaction is a typical chemotropic response, mediated by volatile substances conceivably 
secreted by the zygophores themselves (Banbury, 1954). It is Banbury's opinion that besides the mutual attraction between zygophores of unlike mating type, there also exists a repulsive action between zygophores of the same type, but this has never been confirmed. Extensive work was published about zygotropism by Plempel (1960; 1962) and Plempel \& Dawid (196I) who stated that the hormones involved in zygotropism are not the same as those inducing zygophores.

In this report we describe results which support the statement made by Burgeff (I924) and which indicate that zygophore induction may be brought about not only by trisporic acids but also by volatile factors. They suggest a parallelism between volatile zygophoreinducing and zygotropic action; zygophore induction and zygotropism might even be mediated by the same hormonal factors.

\section{METHODS}

Organisms. The following strains were used: Mucor mucedo Brefeld, 'plus' (CBS I 24.24); M. mucedo Brefeld, 'minus' (CBS 109.16); Blakeslea trispora Thaxter, 'plus' (NRRL 2456); B. trispora Thaxter, 'minus' (NRRL 2457). All strains were obtained from the Centraalbureau voor Schimmelcultures, Baarn, The Netherlands.

Cultures. Mucor mucedo was grown in Petri dishes ( $10 \mathrm{~cm}$ diameter) containing about $\mathrm{I} 5 \mathrm{ml}$ of $20 \%(\mathrm{v} / \mathrm{v})$ beer wort obtained from a local brewery, neutralized to $\mathrm{pH} 8$ with $\mathrm{KOH}$ before sterilization. When appropriate, trisporic acids (mixture of trisporic acids $\mathrm{B}$ and C) were added to the culture medium, to a final concentration of about $15 \mu \mathrm{g} / \mathrm{ml}$. This resulted in mycelia densely covered with relatively short zygophores. After a culture period of 3 to 4 days at $20^{\circ} \mathrm{C}$ in the dark, the mycelial mats were removed with two pairs of sterile forceps, as shown in Fig. I. The folded mat was laid down on one side of a slit cut in a solid substrate $(2 \%, \mathrm{w} / \mathrm{v}$, aqueous agar) in a Petri dish. By doing this, 'plus' and 'minus' mycelia were deposited facing each other, without diffusion contact via the substrate, with a minimum of manipulation. In order completely to eliminate the possibility of diffusion contact, the bottom of the plastic Petri dish was sometimes cut away under the slit. This never had any effect on the observed sexual reactions.

Cultures for bioassay of zygophore induction and zygotropism were grown on a solid glucose-nitrate medium (van den Ende, I968).

Isolation procedures. Neutral zygophore-inducing substances were isolated from the liquid wort media of 'plus' and 'minus' cultures of Mucor mucedo (grown separately) by extraction from a total volume of $300 \mathrm{ml}$ with peroxide-free diethyl ether after adjusting the $\mathrm{pH}$ to $9 \cdot 0$. The extract was washed several times with $5 \%(\mathrm{w} / \mathrm{v})$ aqueous sodium bicarbonate and water (to remove traces of trisporic acids), dried over anhydrous sodium sulphate, and subjected to thin-layer chromatography; $20 \times 20 \mathrm{~cm}$ plates, $0.25 \mathrm{~mm}$ thick, of silica gel H (Merck 7736) were used. The solvent system consisted of chloroform + diisopropyl ether + acetone + acetic acid (70:1 $5: 10: 5$, by vol.). After development, the chromatograms were divided into ten equal zones which were scraped off and eluted twice with ethanol. The solvent in the eluates was removed in vacuo and the residues taken up in I $\mathrm{ml}$ water or ether.

Neutral zygophore-inducing substances were also isolated from Blakeslea trispora cultures as described by Werkman \& van den Ende (1973).

Trisporic acids B and C, purified by chromatography over DEAE Sephadex, were obtained from Blakeslea trispora cultures as described earlier (van den Ende, Werkman \& van den Briel, 1972). 

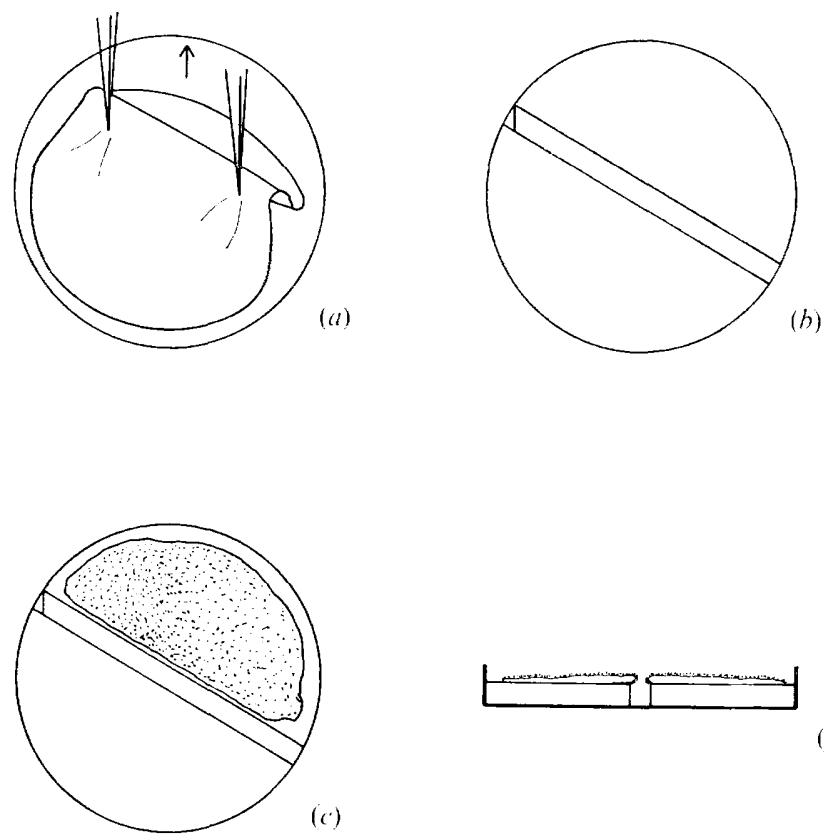

(d)

(c)

Fig. I. Folding and positioning of a mycelial mat on one side of a slit in an aqueous agar substrate.

\section{RESULTS AND DISCUSSION}

Evidence for volatile zygophore-inducing and zygotropic factors

Fig. $2(a-d)$ shows Mucor mucedo at different times during one experiment. Fig. $2(a)$ shows the situation directly after the positioning of a 'plus' and a 'minus' mycelium, both covered with zygophores after growth in a medium containing trisporic acids, facing each other on either side of a slit in an agar layer. Zygophores are collapsed on account of the rather drastic change in microclimate. After $\mathrm{I} 2 \mathrm{~h}$ erect zygophores are visible in large numbers, growing horizontally towards each other (Fig. $2 b$ ). Their length increases considerably, which is an additional feature of the zygotropic reaction (Plempel, I962). Fig. $2(c)$ shows the situation after $20 \mathrm{~h}$. Long zygophores (approx. $\mathrm{I} \cdot 5 \mathrm{~mm}$ ) of both 'plus' and 'minus' mycelia touch each other. The thigmomorphotic reaction after the contact of both mating types" is shown in Fig. $2(d)$.

From a large number of observations of the interactions between both types of mycelium of Mucor mucedo, the following conclusions can be drawn: (I) Zygophores are induced when two unlike vegetative mycelia (grown in the absence of trisporic acids) are on either side of the agar slit. This suggests that there are volatile zygophore-inducing substances other than the trisporic acids produced by vegetative mycelia. (2) When one of the two mycelia is vegetative, the other being covered with zygophores, these will grow in the direction of vegetative mycelium of the other mating type in a horizontal orientation, long before zygophores are visible on the latter. It may be concluded that the volatile zygotropic stimulus is secreted not only by zygophores, but also by vegetative mycelium. Fig. $2(e)$ shows the special case where three 'minus' zygophores are growing in the direction of a 'plus' sporangiophore. (3) No repulsion has ever been observed between zygophores of the same mating type. Sometimes two or more zygophores grow spiralling around each other. When 

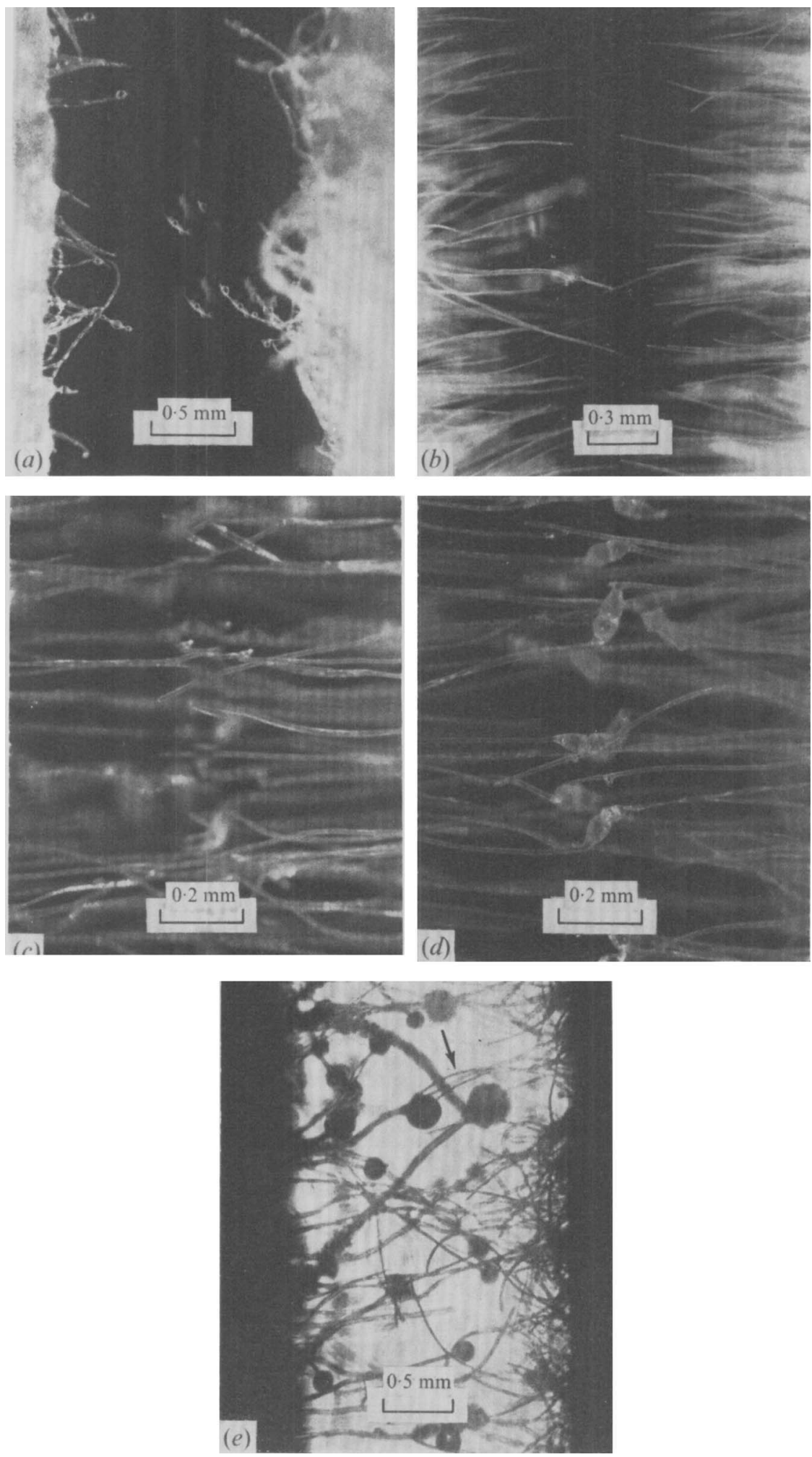

Fig. 2. Sexual reactions of Mucor mucedo, 'plus' and 'minus', positioned on either side of a slit in an aqueous agar substrate (left 'plus', right 'minus'). (a) Zero time, directly after positioning the mycelia; (b) $12 \mathrm{~h}$ later; $(c) 20 \mathrm{~h}$ later; $(d) 24 \mathrm{~h}$ later; (e) zygophores growing in the direction of a sporangiophore (arrow). 
Table I. Zygophore-inducing activity of fractions derived from culture media of Mucor mucedo

\begin{tabular}{|c|c|c|c|}
\hline \multirow[b]{2}{*}{ Fraction tested* } & \multirow{2}{*}{$\begin{array}{c}\text { Test strain of } \\
M . \text { mucedo }\end{array}$} & \multicolumn{2}{|c|}{ Zygophores induced $\uparrow$} \\
\hline & & via air & via substrate \\
\hline Crude "plus" extract & 'plus' & - & - \\
\hline Idem & 'minus' & + & + \\
\hline Idem? & 'plus' & - & - \\
\hline Idem & 'minus' & ++ & ++ \\
\hline Idem $R_{F}=0.0-0.5$ & 'minus' & - & - \\
\hline$R_{F}=0.5-0.7$ & 'minus' & ++ & $t+$ \\
\hline$R_{F}=0.7-1.0$ & 'minus' & - & - \\
\hline Crude 'minus' extract & 'plus' & + & + \\
\hline Idem & 'minus' & - & - \\
\hline Idem: & 'plus' & ++ & ++ \\
\hline Idem & 'minus' & - & - \\
\hline Idem $* R_{F}=0.0-0 . \mathrm{I}$ & 'plus' & $+t$ & ++ \\
\hline$R_{F}=0.1-0.6$ & 'plus' & - & - \\
\hline$R_{F}=0.6-0.8$ & 'plus' & ++ & $+t$ \\
\hline$R_{F}=0.8-1 \cdot 0$ & 'plus' & - & - \\
\hline
\end{tabular}

* $R_{F}$ values on thin-layer chromatograms of ether extracts of culture media. The thin layers were divided into ten zones, which were assayed separately.

$\dagger Z$ ygophore induction via the substrate was accomplished by applying the solution (in water) in a well in the agar. in front of the respective mycelia. Zygophore induction via the air was effected with solutions (in water) in vials positioned on the bottom of the Petri dishes, after cutting away the agar along the front of the mycelium. -, No zygophores after $40 \mathrm{~h} ;+, \mathrm{I}$ to Io zygophores after $40 \mathrm{~h} ;++$, more than 40 zygophores after $40 \mathrm{~h}$.

$\$$ The culture had grown in the presence of trisporic acids.

two mycelia of the same mating type, with zygophores, are positioned on either side of the slit, no reaction whatever can be detected. In due course a metamorphosis of zygophores to sporangiophores is observed. (4) The thigmomorphotic reaction can take place without prior zygotropic reaction. Two unlike mycelia containing zygophores can be positioned so that the zygophores exactly touch each other. The normal cell-fusion reaction is then observed within $2 \mathrm{~h}$.

\section{Isolation of volatile zygophore-inducing and zygotropic substances}

Solid media in Petri dishes were eccentrically inoculated with single strains of Mucor mucedo. After three days the uncovered agar was cut away along the front of the mycelium, and small glass vials ( $4 \mathrm{~mm}$ depth, internal diameter $9 \mathrm{~mm}$ ) were positioned about $4 \mathrm{~mm}$ from the front of the mycelium on the bottom of the dish. The vials were filled with crude ether extracts of liquid culture medium, or fractions thereof dissolved in water. In this way zygophore induction would be mediated unambiguously via the air. As is shown in Table I, zygophores were induced on 'plus' and 'minus' mycelia of $M$. mucedo by fractions obtained from culture media of the sexual partner. The same results were obtained when extracts of culture media of Blakeslea trispora were used. Trisporic acid solutions never gave a response in this type of bioassay. Likewise, controls in which media or extracts were used which were derived from the same strain as used for the bioassay, were always negative. Sometimes, however, poorly reproducible responses were observed when fractions of 'plus' medium were applied to the 'plus' strain of $M$. mucedo. The structures induced had the appearance of zygophores, but without zygotropic features (see below). We tentatively designate these as pseudophores, as described by Plempel (I963). 


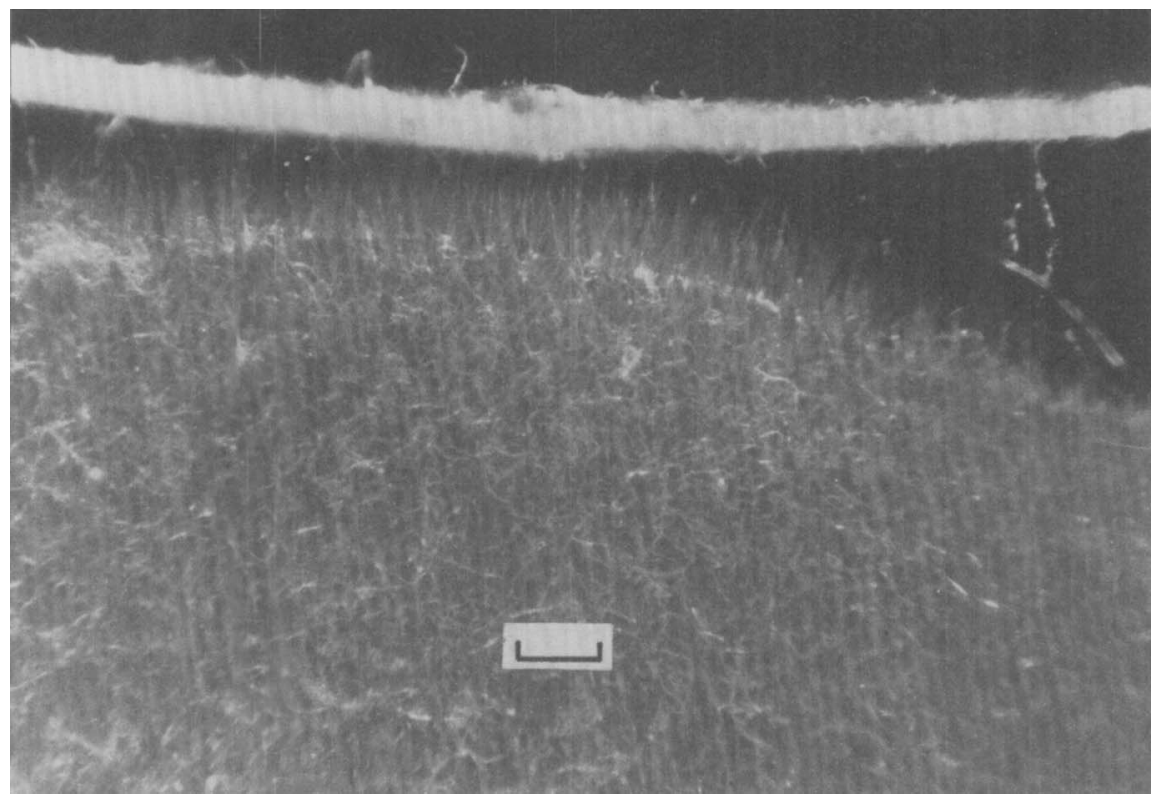

Fig. 3. Orienting effect on zygophores of Mucor mucedo 'plus' by a piece of filter paper impregnated with an ether extract of culture medium of Mucor mucedo 'minus'. The photograph was taken $20 \mathrm{~h}$ after the start of the experiment. Bar marker $=1 \mathrm{~mm}$.

There are two facts to be noted in particular: (i) biological activity was detected in the same fractions as found earlier to contain zygophore-inducing precursors of trisporic acids in Blakeslea trispora (Werkman \& van den Ende, 1973) and Mucor mucedo (B. A. Werkman, unpublished); (ii) biological activity was much higher in fractions derived from cultures treated with trisporic acids and, in addition, 'minus'-culture extracts were more active than 'plus'-culture extracts. This suggests that airborne zygophore induction is mediated by these precursors of trisporic acids, the synthesis of which is stimulated by trisporic acids. Starting with equal culture volumes and keeping all circumstances as similar as possible, 'minus' extracts usually display more vigorous reactions than 'plus' extracts; this could be the result of more abundant production of active substances by the 'minus' strain, or of a higher sensitivity of the 'plus' strain. This at least rationalizes the observation of Gooday (1968) that in normal conjugations the 'plus' strain gives rise to zygophores both earlier and in larger numbers than the 'minus' strain.

All neutral fractions of culture media which induced zygophores, also had a marked effect on their morphology. Zygophores were longer and oriented towards the source of the agent. Fig. 3 shows a piece of filter paper, impregnated with an active fraction, placed in a vertical position near a mycelium containing randomly oriented zygophores induced by trisporic acids via the substrate. The zygophores nearest to the paper are more extended and grow in its direction, whereas the more distant ones keep their more-or-less vertical orientation. This suggests that volatile fractions which actively induced zygophores, also had zygotropic action. Whether we are dealing with substances having both the zygophore-inducing and zygotropic action, or with different (although related) substances exerting these two effects, cannot be decided from these experiments. However, the results do not preclude assigning this chemotropic role to precursors of trisporic acids, as isolated from the substrate. 
The latter suggestion could imply that zygophore induction and zygophore orientation are based on the same mechanism. Bartnicki-Garcia \& Lippman (1972) have suggested that enzymes that open bonds in the hyphal wall, leading to initiation of apical growth (e.g. branching or, more specifically, zygophore formation), are an integral part of the normal growth process. Stimulation and orientation of apical growth of zygophores could be visualized as a (localized) stimulation of these bond-splitting enzymes, thus changing the balance of wall softening and wall synthesis at the tips of the zygophore.

An analogous situation is found in Achlya, where the sex hormone antheridiol initiates the formation of antheridial branches and also elicits from them a chemotropic response (Barksdale, 1969).

Finally, the observation that the biologically active factors described here are not species specific with respect to Mucer mucedo and Blakeslea trispora, nor to M. hiemalis (unpublished), may provide the physiological basis for the understanding of the well-known interspecific conjugations which have been described by Burgeff (I924) and Blakeslee \& Cartledge (1927).

We acknowledge the active interest of J. F. L. M. Urbanus.

\section{REFERENCES}

BANBURY, G. H. (I954). Processes controlling zygophore formation and zygotropism in Mucor mucedo Brefeld. Nature, London 173, 499-500.

Barksdale. A. W. (1969). Sexual hormones of Achlya and other fungi. Science, Washington I66, 83I-837.

BARTNICKI-GARCIA, S. \& LiPPMAN, E. (1972). The bursting tendency of hyphal tips of fungi: presumptive evidence for a delicate balance between wall synthesis and wall lysis in apical growth. Journal of General Microbiology 73, 487-500.

BlakesleE, A. F. \& CARTLedge, J. L. (1927). Sexual dimorphism in Mucorales. II. Interspecific reactions. Botanical Gazette 84, 5 I-57.

Bu'LoCK, J. D. \& WinstanLEY, D. J. (197I). Carotenoid metabolism and sexuality in Mucorales. Journal of General Microbiology 68, xvi-xvii.

BurgefF. H. (1924). Untersuchungen über Sexualität und Parasitismus bei Mucorineen. I. Botanische Abhandlungen 4, I-I 35 .

VAN DEN ENDE, H. (1968). Relationship between sexuality and carotene synthesis in Blakeslea trispora. Journal of Bacteriology 96, I $298-1303$.

van den Ende, H. \& Stegwee, D. (1971). Physiology of sex in Mucorales. Botanical Review 37, 22-36.

VAN den Ende, H., Werkman, B. A. \& van den Briel, M. L. (1972). Trisporic acid synthesis in mated cultures of the fungus Blakeslea trispora. Archiv fïr Mikrobiologie 86, I75-184.

Gooday, G. W. (1968). Hormonal control of sexual reproduction in Mucor mucedo. New Phytologist 67, $8 \mathrm{I} 5-821$.

Gooday, G. W. (1973). Differentiation in the Mucorales. Symposium of the Society for General Microbiology. 23, 269-294.

MACHLis, L. (1973). The coming of age in sex hormones in plants. Mycologia 64, 235-247.

Plempel, M. (1960). Die zygotropische Reaktion bei Mucorineen. I. Planta 55, 254-258.

Plempel, M. (1962). Die zygotropische Reaktion bei Mucorineen. III. Planta 58, 509-520.

Plempel, M. (1963). Die chemischen Grundlagen der Sexualreaktion bei Zygomyceten. Planta 59, 492-508,

Plempel, M. \& Dawid, W. (I961). Die zygotropische Reaktion bei Mucorineen. II. Planta 56, 438-446.

Sutter, R. P. (1970). Trisporic acid synthesis in Blakeslea trispora. Science, Washington 168, 1590-1592.

Sutter, R. P., Capage, D., Harrison, T. L. \& Keen, W. A. (1973). Trisporic acid biosynthesis in separate plus and minus cultures of Blakeslea trispora: identification by Mucor assay of two mating-type specific components. Journal of Bacteriology 114, 1074-1090.

Werkman. B. A. \& VAN Den ENDE, H. (I973). Trisporic acid synthesis in Blakeslea trispora. Interactions between plus and minus mating types. Archiv fiir Mikrobiologie 9o, 365-374. 